\title{
Article \\ Optimal Assistance Timing to Induce Voluntary Dorsiflexion Movements: A Preliminary Study in Healthy Participants
}

\author{
Jing-Chen Hong $1, * \mathbb{D}$, Kazuhiro Yasuda ${ }^{2}$, Hiroki Ohashi ${ }^{3}$ and Hiroyasu Iwata ${ }^{4}$ \\ 1 Graduate School of Creative Science and Engineering, Waseda University, Tokyo 169-8050, Japan \\ 2 Research Institute of Science and Engineering, Waseda University, Tokyo 169-8050, Japan; \\ kazuhiro-yasuda@aoni.waseda.jp \\ 3 Department of Neurosurgery, Jikei University School of Medicine, Tokyo 105-8461, Japan; \\ hirokioha@gmail.com \\ 4 Faculty of Science and Engineering, Waseda University, Tokyo 169-8050, Japan; jubi@waseda.jp \\ * Correspondence: charles4543@toki.waseda.jp; Tel.: +81-3-3203-4427
}

Citation: Hong, J.-C.; Yasuda, K.; Ohashi, H.; Iwata, H. Optimal Assistance Timing to Induce Voluntary Dorsiflexion Movements: A Preliminary Study in Healthy Participants. Appl. Sci. 2022, 12, 2248. https://doi.org/10.3390/ app12042248

Academic Editor: Philip Fink

Received: 20 January 2022

Accepted: 17 February 2022

Published: 21 February 2022

Publisher's Note: MDPI stays neutral with regard to jurisdictional claims in published maps and institutional affiliations.

Copyright: (C) 2022 by the authors. Licensee MDPI, Basel, Switzerland. This article is an open access article distributed under the terms and conditions of the Creative Commons Attribution (CC BY) license (https:// creativecommons.org/licenses/by/ $4.0 /)$.

\begin{abstract}
Swing-phase dorsiflexion assistance with robotic ankle-foot orthosis could improve toe clearance and limb shortening such that compensatory movements are suppressed. However, facilitating voluntary effort under assistance remains a challenge. In our previous study, we examined assistance effects of swing-phase dorsiflexion with different delay times after toe-off on a dorsiflexionrestricted gait with a high-dorsiflexion assistive system. Results showed that later dorsiflexion assistance could lead to an increase in the tibialis anterior's surface electromyography but could also deteriorate compensatory movement. Thus, we concluded that there is a suitable assistance timing to simultaneously achieve voluntary effort and optimal gait. In the present research, we derived a method to identify a suitable dorsiflexion assistance delay time via a multiple linear regression analysis on ankle data of stroke patients with a pathological gait with insufficient dorsiflexion. With the identification method, an experiment was conducted on six healthy participants with restricted dorsiflexion. Results showed that the identified assistance timing improved the amplitude of the tibialis anterior's surface electromyography while also suppressing limb shortening during circumduction and hip hiking. Although a practical study of stroke survivors is required, observations from this research indicate the potential to successfully induce voluntary efforts with the identified dorsiflexion assistance timing.
\end{abstract}

Keywords: robotic ankle-foot orthosis; assist-as-needed; voluntary effort; compensatory movement; limb shortening

\section{Introduction}

Stroke is presently one of the most prevalent cardiovascular diseases. According to the World Health Organization, it is estimated that 15 million people worldwide suffer a stroke annually, 10 million of whom survive with disabilities [1]. Treatment, rehabilitation, and caregiving of the stroke survivors could lead to a large financial and societal burden [2]. One critical tool to address this issue is efficient rehabilitation programs to improve recovery speed, such as the introduction of robotic gait assistance programs to help stroke survivors regain their gait abilities [3].

Hemiplegia that leads to paralysis of the affected side is a common sequela for stroke survivors [4]. For a normal human gait, dorsiflexion in the swing phase contributes to limb shortening, which is important to ensure toe clearance from the ground [5,6]. For a stroke hemiplegic patient, however, his or her ankle forces or movement controls may be too weak to perform sufficient dorsiflexion and drop foot could result [7]. This toe-down posture decreases toe clearance and could lead to stumbling or toe dragging while walking [8]. To increase minimum toe clearance (MTC) in the swing phase, stroke survivors tend to 
perform compensatory movements such as circumduction and hip hiking $[9,10]$. However, such compensatory movements during gait rehabilitation could affect optimal gait learning.

Robotic ankle-foot orthoses (RAFOs) have been developed in recent decades to support ankle movements during gait rehabilitation, including dorsiflexion assistance in the swing phase [11,12]. Some studies have indicated that their RAFOs' intervention effects not only improve ankle dorsiflexion angle and foot clearance but also suppress compensatory movements $[12,13]$. There are also off-the-shelf RAFO systems, such as RE-Gait ${ }^{\circledR}$, currently used in robotic gait assistance programs in medical facilities [14].

Although promising intervention results were reported in the available literature, there are still challenges left for RAFO technology, such as the implementation of an assistas-needed (AAN) control strategy. Robotic systems such as Lokomat and ALEX apply impedance control to enhance voluntary lower-limb movements during gait rehabilitation [15-17]. It is also commonly held that increasing challenge and voluntary effort during a rehabilitation program accelerates recovery for stroke survivors $[15,18]$. Current RAFOs mostly apply positional control, force control, or event-trigger control for ankle movement assistance [19]. Such control strategies purposefully introduce difficulty in movement to further induce a patient's voluntary effort during rehabilitation. Anklebot applies impedance control to provide suitable assistance for ankle movement control [20]. However, most applications are conducted in a sitting posture with the heel on the ground. The AAN control for ankle movement while walking is required, and realization of an AAN strategy on swing-phase dorsiflexion is one of our ultimate goals.

Another gap is a systematic gait analysis to verify the RAFO's assistance effects. Current gait measurement instruments can already carry out a systematic gait analysis. An example is an easy gait analysis system that could analyze spatiotemporal gait parameters with Kinect [21]. For lower-limb exoskeletons, a study has assessed not only Lokomat's intervention effect on lower-limb kinematics but also lower-limb range of motion and stiffness which are also important to track a stroke survivor's recovery status [22]. For RAFO research, Anklebot could analyze ankle stiffness [23]. However, to our understanding, very few or no studies have reported systematic gait analysis with an RAFO's assistance. On the other hand, studies on conventional ankle-foot orthoses have reported intervention effects on not only ankle kinematics but also previously mentioned parameters such as MTC and circumduction [24]. Assistance with ankle movements could affect overall gait patterns, and kinematics in individuals should be different. Therefore, analysis on the targeted joint alone is not sufficient to verify an assistive system's feasibility.

In our previous study, the potential to use a delayed-timing activation of dorsiflexion assistance to improve a user's voluntary effort was investigated and found feasible [25]. We have developed a high-dorsiflexion assistance system that assists swing-phase dorsiflexion upon toe-off with a pneumatic artificial muscle, and a pilot study has shown its intervention effects to improve ankle dorsiflexion angle [26]. However, voluntary dorsiflexion could be hindered with the instant activation of dorsiflexion assistance at toe-off [27]. Seeking to improve voluntary efforts while ensuring optimal gait without compensatory movements, we conducted a trial on healthy participants with dorsiflexion-restricted gaits to investigate different delay times after toe-off [25]. The results show that longer assistance delay times had moderate to strong positive correlations with surface electromyography (sEMG) of the tibialis anterior, indicating that delayed assistance could increase the extent of voluntary dorsiflexion movement. On the other hand, positive correlation was also found between assistance delay times and extent of compensatory movements, which indicates that later assistance could also deteriorate gait pattern. Thus, modulating assistance timing can serve as a method to adjust assistance level, but a trade-off between the extent of voluntary effort and extent of compensatory movement is present. Our findings indicate that there is a unique assistance delay time for an individual that maximizes voluntary efforts while suppressing compensatory movements.

In this present research, we propose a method to identify an optimal delay time to activate dorsiflexion assistance from our high-dorsiflexion assistive system. Linear multiple 
regression was used to derive an identification equation by analyzing ankle angle data from stroke survivors. A test was conducted on six healthy participants with restricted swing-phase dorsiflexion to verify the effects of the derived equation. In addition to dorsiflexion angle, effects on the sEMG of the tibialis anterior and the extent of compensatory movements were also analyzed to verify feasibility of the identified timing. It is expected that the identified assistance timing would enhance the participants' voluntary efforts compared to assistance without delay while also preventing compensatory movements such as circumduction and hip hiking.

\section{Materials and Methods}

\subsection{The High-Dorsiflexion Assistive System}

Figure 1a shows the high-dorsiflexion assistive system, and Figure $1 \mathrm{~b}$ depicts the configuration of the system [25]. Insoles included force sensors (Flexiforce A301 Sensor, Tekscan Inc., South Boston, MA, USA) to detect grounding status of the first metatarsal bone, which was reported as the position with the largest ground reaction force at push-off. Through a wireless module (nRF24L01, Nordic Semiconductor, Trondheim, Norway), the force sensor data were transmitted to the microcontroller, also with an embedded wireless module (nRF52832, Nordic Semiconductor, Norway). The microcontroller transmits signals through a wired connection to solenoid valves (BV214A, MAC Valves Inc., Wixom, MI, USA) that control air supply and release to the artificial muscle tube (DMSP-10, Festo Inc., Esslingen am Neckar, Baden-Württemberg, Germany). A portable cylinder with $74 \mathrm{~g}$ compressed carbon dioxide was used as the air source for the system. For easy execution, we used a stationary air compressor with a 30 L tank (SW-231, Fujiwara Sangyo Co., Ltd., Hyogo, Japan). A tension spring (Tokai Spring Industries Inc., Osaka, Japan) was placed in series with the artificial muscle between the knee and forefoot. Parameters of the system including assistance activation delay time were manually set with a selfdeveloped graphical interface, and the manipulation signal was transmitted wirelessly to the microcontroller. The lower limb is only $0.35 \mathrm{~kg}$ while providing up to $75 \mathrm{Nm}$ dorsiflexion torque assistance. Previous research suggests that an external torque of $13.5 \mathrm{Nm}$ is required to assist ankle dorsiflexion from foot drop to a normal posture [28]. Therefore, our system could fully assist swing-phase dorsiflexion even with no dorsiflexion ability.

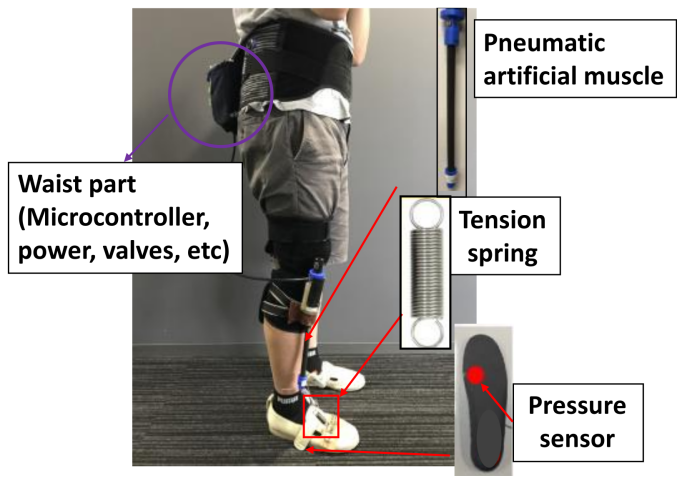

(a)

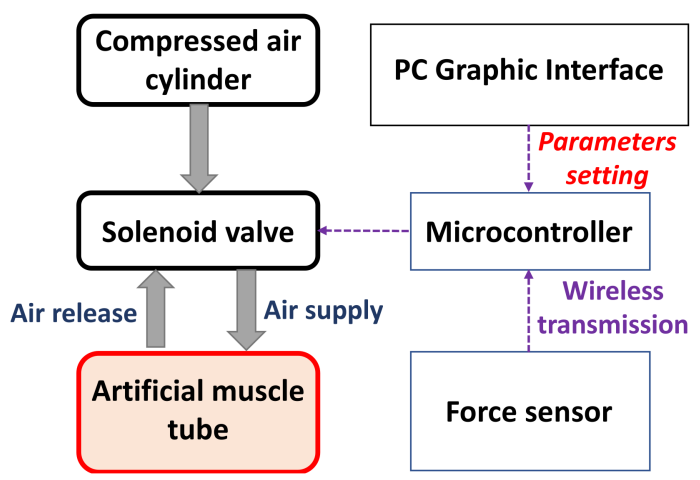

(b)

Figure 1. The high-dorsiflexion assistive system: (a) Photograph of the system; (b) system configuration.

Figure 2a depicts the control diagram, and Figure 2b depicts the system's assistance in one gait cycle. Upon toe-off, no ground reaction force is sensed by the force sensor, and the artificial muscle compresses to assist dorsiflexion during the whole swing phase. From heel contact to midstance of gait, the artificial muscle keeps contracting while the tension spring extends and provides a resistive dorsiflexion torque, which assists the heel rocker function during the loading response phase [29]. After the midstance phase, the ground reaction force at the forefoot increases and the artificial muscle extends until the next toe-off. In this research, we focus on the swing-phase dorsiflexion assistance provided by the artificial 
muscle. Using the graphical interface, the activation of dorsiflexion assistance was timed

based on the specified value in milliseconds after toe-off.

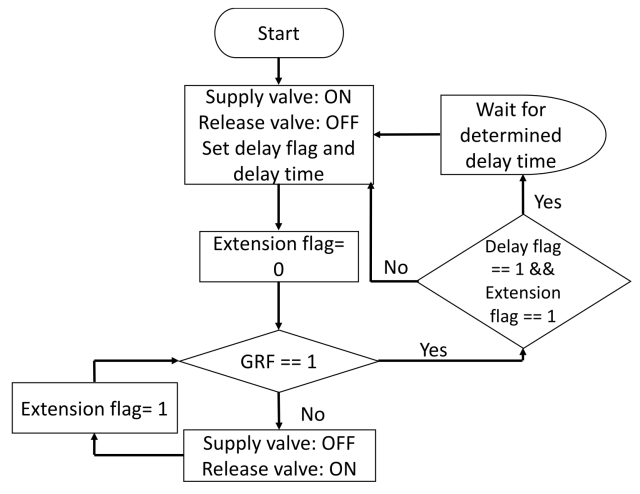

(a)

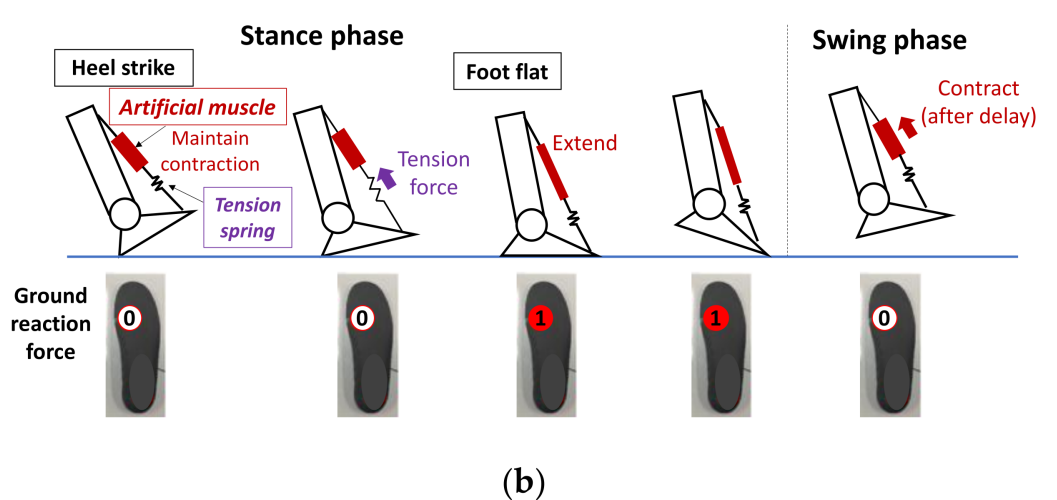

(b)

Figure 2. (a) Control diagram; (b) system control in a gait cycle.

\subsection{Identification of a Suitable Assistance Delay Time}

Figure 3 depicts ankle angle in a gait cycle with an assumed case of insufficient ankle dorsiflexion on the paralyzed side of a stroke survivor. The ankle dorsiflexion angle in the swing phase on the paralyzed side is typically smaller than that on the healthy side. However, the difference between the paralyzed and healthy sides varies between participants, possibly due to recovery status. An ideal assistance timing that ensures voluntary movement before assistance and makes up for an insufficient dorsiflexion angle is labeled in the figure. An excessively early assistance could hinder voluntary effort, while an excessively late assistance could decrease MTC or force compensatory movements.

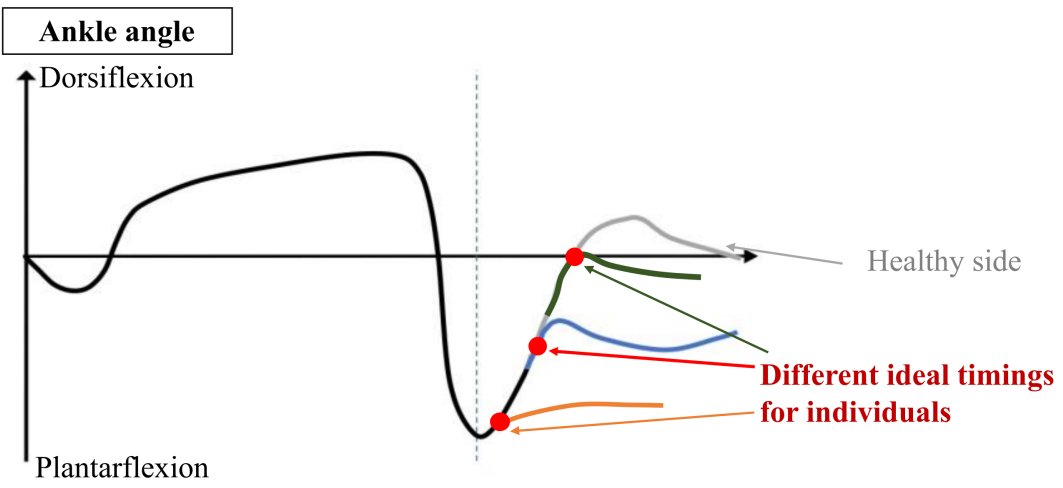

Figure 3. Assumed figure of ankle angle data for a stroke survivor with insufficient swing-phase dorsiflexion.

From the previous assumption, we aim to derive an equation to identify an ideal assistance timing that simultaneously ensures voluntary dorsiflexion movement and prevents compensatory movements. Ankle angle data to derive an identification equation were obtained from medical records from 2017 to 2020 in our previous studies on a vibrotactile system [30-33]. The data were collected with a portable motion gait analysis system (RehaGait, HASOMED GmbH, Magdeburg, Saxony-Anhalt, Germany) [34]. In some of these studies, a vest with vibrators was used to provide biofeedback on toe-off and heel contact events to stroke survivors who can walk individually with or without canes [30,31]. Improvements in swing-phase dorsiflexion and push-off angles were observed during and after intervention. In the other studies [32,33], a waist belt with vibrators was used to provide cadence cueing. With implicitly increasing cadence cued to the stroke survivors, their walking speed increased without worsening of gait kinematics. Ankle angle data 
before intervention from 16 of the stroke participants (Brunnstrom's stages V-VI) whose paralyzed sides suffer insufficient dorsiflexion in the swing phase were selected. Data from 11 of these participants were used for the multiple linear regression analysis, and the others for verification and validation. As Figure 4 indicates, the ideal assistance timing was set when the angular acceleration equaled zero (i.e., the second derivative of angle data equaled zero) after toe-off, and the assistance delay $y$ was the time from toe-off to the assistance timing activation. With the ankle angle data, multiple regression was used to test whether the explanatory variables in Figure $4\left(x_{1}, x_{2}, x_{3}, x_{4}\right)$ could significantly predict the suitable assistant delay time $y$ in milliseconds. The regression equation for assistance delay is as follows:

$$
y=A x_{1}+B x_{2}+C x_{3}+D x_{4}+E
$$

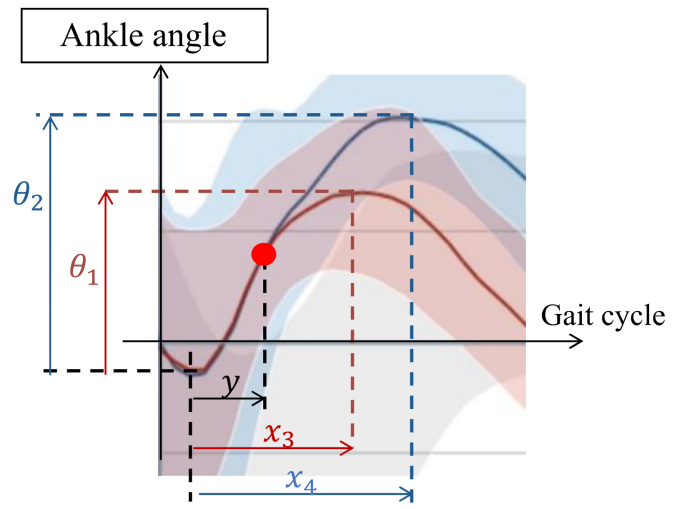

$x_{1}$ : Gait cycle $(\mathrm{ms})$

$x_{2}$ : Ratio of dorsiflexion angle at both side $\frac{\theta_{1}}{\theta_{2}}$

$x_{3}$ : Gait cycle ratio for dorsiflexion at paralyzed side

$x_{4}$ : Gait cycle ratio for dorsiflexion at healthy side

Figure 4. Ankle angle with assumed ideal assistance timing and explanatory variables.

The results of the multiple linear regression are shown in Table 1. According to the results, gait cycle ratios of duration from toe-off to the maximum dorsiflexion angle at the healthy side $\left(x_{4}\right)$ did not significantly predict the delay time for activating dorsiflexion assistance after toe-off $(y)$. The multiple linear regression analysis was repeated using only the remaining three explanatory variables, and the results are shown in Table 2 . The identification equation for delay time for optimal dorsiflexion assistance was derived as follows:

$$
y=-0.089 x_{1}+58.87 x_{2}+711.811 x_{3}+42.031\left(p<0.05, R^{2}=0.74\right)
$$

Table 1. Multiple linear regression analysis results to identify optimal assistance delay time.

\begin{tabular}{cccc}
\hline Explanatory Variable & Coefficient & $\begin{array}{c}\text { Significance } \\
\text { Judgment }^{\mathbf{1}}\end{array}$ & VIF $^{\mathbf{2}}$ \\
\hline$x_{1}$ & -0.0925 & $*$ & 1.087 \\
$x_{2}$ & 57.407 & $*$ & 1.062 \\
$x_{3}$ & 874.31 & $*$ & 2.728 \\
$x_{4}$ & -173.38 & & 2.755 \\
constant & 56.643 & - & - \\
\hline
\end{tabular}

$1 *: p<0.05 ;^{2}$ VIF, variance inflation factor. 
Table 2. Multiple linear regression analysis results excluding $x_{4}$, which did not significantly predict assistance delay time in the previous analysis.

\begin{tabular}{cccc}
\hline Explanatory Variable & Coefficient & $\begin{array}{c}\text { Significance } \\
\text { Judgment }^{\mathbf{1}}\end{array}$ & VIF $^{2}$ \\
\hline$x_{1}$ & -0.089 & $*$ & 1.087 \\
$x_{2}$ & 58.87 & $*$ & 1.062 \\
$x_{3}$ & 711.811 & - & 2.728 \\
constant & 42.031 & & - \\
\hline $1 *: p<0.05 ;{ }^{2}$ VIF, variance inflation factor.
\end{tabular}

Data from the other five stroke survivors with insufficient swing-phase dorsiflexion were used for verification and validation. The predicted delay time values were calculated using Equation (2), and the real values were determined as the duration from toe-off to the time stamp where the second derivative of the ankle angle curve equaled zero. The results are shown in Table 3. The maximum error between the real and predicted values is approximately $3 \mathrm{~ms}$. From the results, we conclude that the identification equation can accurately determine the ideal assistance timing of swing-phase dorsiflexion.

Table 3. Verification and validation results for the multiple linear regression model.

\begin{tabular}{cccc}
\hline Patient & Suitable Delay Time $(\mathbf{m s})$ & $\begin{array}{c}\text { Predicted Delay } \\
\text { Time }(\mathbf{m s})\end{array}$ & Error (ms) \\
\hline A & 88.16 & 85.94 & $-2.22(-2.52 \%)$ \\
B & 123.36 & 125.17 & $1.81(1.47 \%)$ \\
C & 98.56 & 95.59 & $-2.97(-3.01 \%)$ \\
D & 101.32 & 98.15 & $-3.17(3.13 \%)$ \\
E & 98.00 & 100.05 & $2.05(2.09 \%)$ \\
\hline
\end{tabular}

The identification procedure is expected to be simple even in real medical facilities. Before using our high-dorsiflexion assistive system, the gait data from the patient are measured with Rehagait. From Rehagait's analysis, the explanatory variables $x_{1}, x_{2}$, and $x_{3}$ can be easily accessed. Lastly, using Equation (2), the ideal assistance delay time for swing-phase dorsiflexion can be determined. The parameter can then be set by our system through the self-made graphic interface.

\section{Experiment}

With the identified delay time of dorsiflexion assistance, the next goal is to suppress compensatory movements while facilitating voluntary dorsiflexion movements. An experiment on healthy participants with dorsiflexion-restricted gaits was conducted and evaluated whether the results with the identified assistance timing contributed to improved performance.

\subsection{Participants}

Participants in this experiment were six healthy individuals whose basic information is summarized in Table 4. All of them were assumed paralyzed on the right side. Their dorsiflexion movements were restricted by an orthosis with a tension spring placed above the right calf while walking, as shown in Figure 5. With swing-phase dorsiflexion restricted, the participants displayed compensatory movements to prevent toe dragging and stumbling. In our previous study, we found that healthy participants could perform a simulated steppage gait by excessively lifting their knee in the swing phase or simulate a circumduction gait by swinging their leg outward [25]. Due to weak hip and knee flexion movements in most stroke survivors, there are more reports of circumduction gaits than steppage gaits. Therefore, we selected the six participants who exhibited a simulated circumduction gait while their swing-phase dorsiflexion was restricted for this research. 
Table 4. Information of the participants.

\begin{tabular}{ccccc}
\hline Participant & Gender & Age (Years) & Height (cm) & Weight (kg) \\
\hline A & Male & 26 & 170 & 70 \\
B & Female & 24 & 163 & 53 \\
C & Male & 24 & 172 & 102 \\
D & Female & 23 & 166.5 & 50.5 \\
E & Male & 25 & 175 & 100 \\
F & Male & 32 & 172 & 76 \\
\hline
\end{tabular}
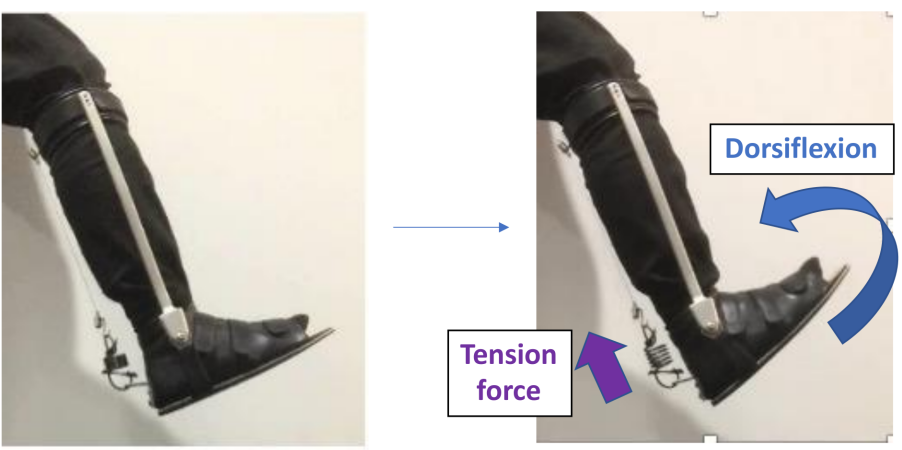

Figure 5. The dorsiflexion-restricted orthosis.

\subsection{Experimental Design}

The conditions of this experiment were as follows:

- Simulated circumduction gait (SCG): The participants walked with the dorsiflexionrestricted $\mathrm{AFO}$, and an extension spring was installed to restrict swing-phase dorsiflexion. Thus, they could simulate a circumduction gait.

- Assistance without delay (AST0): The participants walked with restricted dorsiflexion, and our high-dorsiflexion assistive system activated dorsiflexion assistance upon toe-off.

- $\quad$ Assistance with delay time of $0.5 \mathrm{y}, \mathrm{y}, 1.5 \mathrm{y}$ ms (AST0.5y, ASTy, AST1.5y): The participants walked with restricted dorsiflexion, and the high-dorsiflexion assistive system activated after a $0.5 \mathrm{y}, \mathrm{y}, 1.5 \mathrm{y}$ ms delay. The sequences of these three conditions were random for all participants.

Under these conditions, the participants were requested to walk $5 \mathrm{~m}$ six times. Before evaluating under a new condition, the participants were requested to practice for at least $3 \mathrm{~min}$. The previous study reported a strong positive correlation between assistance delay time and sEMG results and between assistance time and the extent of compensatory movements [25]. Therefore, in addition to the identified assistance delay time y (ASTy), a significantly longer delay time (AST1.5y) and a significantly shorter delay time (AST0.5y) were selected for statistical comparison.

\subsection{Measurement and Evaluation}

The physiological data were set as the sEMG of the tibialis anterior, measured by a Trigno $^{\mathrm{TM}}$ Wireless System (Delsys Inc., Natick, MA, USA) sampled at $2000 \mathrm{~Hz}$. The data were analyzed by EMG works (Delsys Inc., Natick, MA, USA). According to the studies on lower-limb sEMG processing and analysis [35,36], the collected data were filtered with a fourth-order Butterworth bandpass filter, and cut-off frequencies were set between 20 and $450 \mathrm{~Hz}$. Before the experiment, each participant's maximum voluntary contraction (MVC) was collected. After filtering, the MVC data were processed by root mean square with a $300 \mathrm{~ms}$ movable window. The collected data in the experiment were filtered and processed by amplitude analysis, and the evaluation index was set as the maximum percentage referencing the processed MVC data during the swing phase. The index was regarded as the extent of voluntary dorsiflexion. 
The kinematic data were measured and collected with a motion capture system (MAC3D, NAC Inc., Japan) consisting of eight high-speed cameras. The sampling rate for data collection was set at $200 \mathrm{~Hz}$. Figure 6a shows a photo of the reflection markers placed on a participant's body, and Figure $6 \mathrm{~b}$ illustrates the marker setting:

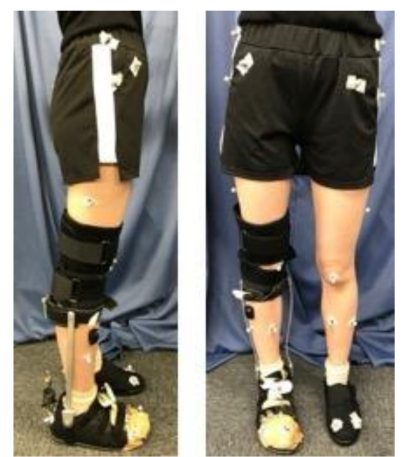

(a)

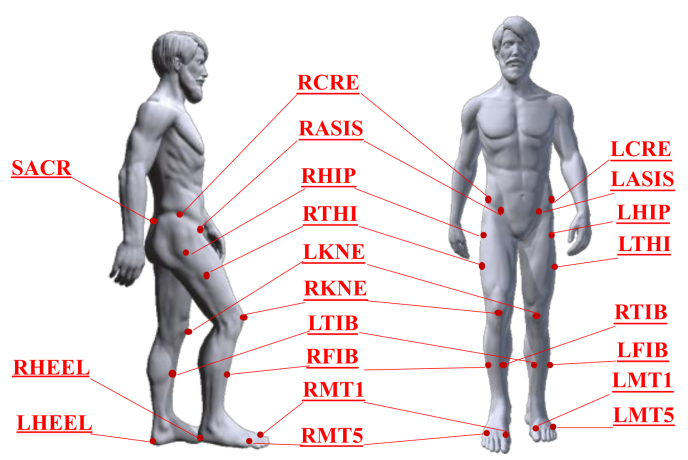

(b)

Figure 6. (a) A photograph of markers placed on a participant; (b) the marker set in this research.

- $\quad$ RHEEL, LHEEL: Heels on both feet;

- $\quad$ RMT1, LMT1: First metatarsal bones in both feet;

- $\quad$ RMT5, LMT5: Fifth metatarsal bones in both feet;

- $\quad$ RTIB, LTIB: Middle of tibias of both legs;

- RFIB, LFIB: Middle of fibulas of both legs;

- $\quad$ RKNE, LKNE: Patellas of both legs;

- $\quad$ RTHI, LTHI: Middle of lateral thighs of both legs;

- RHIP, LHIP: Greater trochanter of both legs;

- RCRE, LCRE: Iliac crests of both sides;

- $\quad$ RASIS, LASIS: Anterior superior iliac spines of both sides;

- SACR: Sacrum.

After low-pass filtering with a cut-off frequency at $6 \mathrm{~Hz}$, the recorded data were analyzed by nMotion musculous (NAC Inc., Tokyo, Japan) to derive gait parameters including lower-limb joint angles. Due to the placement of the dorsiflexion-restricted AFO, the markers could not be placed at the ankle joints. With nMotion musculous's lower-limb model, the ankle joint angles could still be calculated with the markers set from toe to knee.

Evaluation indices for kinematic data include swing-phase dorsiflexion angle, limb shortening in the swing phase, swing width, and hip hike. Swing-phase dorsiflexion angles were calculated by nMotion musculous. The other indices are illustrated in Figure 7. Limb shortening was defined as the decrease in distance between the hip joint to the forefoot in the swing phase compared with that in a standing posture, calculated as the distance between the RHIP and RMT5 markers. Extent of circumduction and hip hiking was evaluated by swing width and hip hike, which are the expected compensatory movements if ankle dorsiflexion or limb shortening in the swing phase is insufficient. Swing width was defined as the RMT1 marker's lateral swing distance in the swing phase, and hip hiking was defined as the RHIP marker's ascent in the swing phase. 

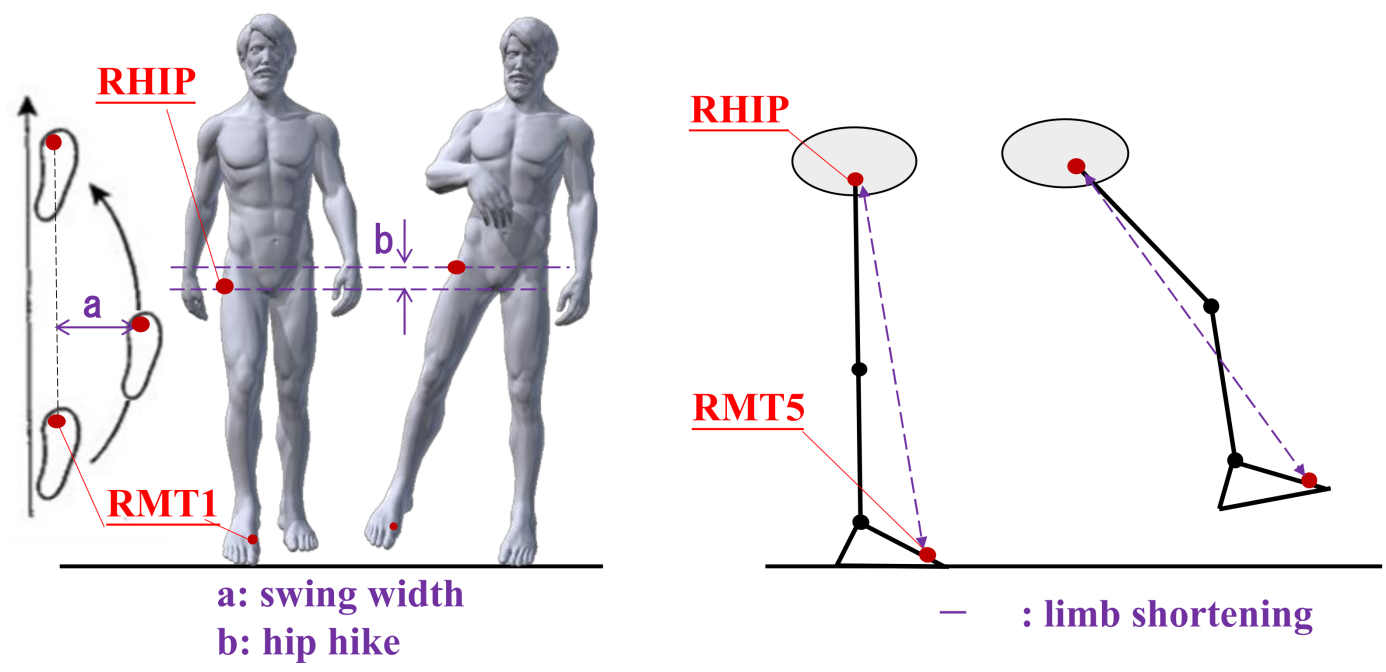

Figure 7. Evaluation indices indicating extent of circumduction, hip hiking, and limb shortening.

\subsection{Statistical Analysis}

The evaluation indices for each individual were analyzed. Our previous work reported significant decreases in sEMG in the tibialis anterior when dorsiflexion was assisted with our system upon toe-off [27], referenced as the AST0 condition in this experiment. Therefore, the physiological index was analyzed to observe whether voluntary dorsiflexion was induced with delayed assistance compared to that with instant assistance. For all participants' processed sEMG results, a Kruskal-Wallis $\mathrm{H}$ test was used to analyze whether there were overall significant differences between conditions AST0, AST0.5y, ASTy, and AST1.5y. If significant differences were observed, a Dunn test with Bonferroni correction was used to compare pair-wise differences between all delayed-time assistance and AST0 conditions.

The other stated goal for our identified optimal dorsiflexion assistance timing is to suppress compensatory movements. That is, a decrease in swing width and hip hike compared with those in the SCG condition was expected. Meanwhile, ankle dorsiflexion angle and limb shortening could affect the extent of compensatory movements $[6,10]$. Thus, the influence of assistance timing on this is also considered. A Kruskal-Wallis $\mathrm{H}$ test was used to compare kinematic indices among all measured conditions. If overall significant differences were observed, a Dunn test with Bonferroni correction was used to compare differences between indices in SCG and those in each assistance condition.

Analysis results of $0.01 \leq p<0.05$ were interpreted as statistically significant, and $p<0.01$ was interpreted as highly statistically significant. Moreover, $0.05 \leq p<0.10$ was interpreted as marginally statistically significant. All analyses were conducted with SPSS 27 (IBM, Armonk, NY, USA).

\section{Results}

\subsection{The Physiological Result}

Physiological index results are shown in Figure 8. Results of the Kruskal-Wallis H tests for all participants are all highly significant. Compared with AST0, the tibialis anterior's sEMG amplitude in the ASTy condition for Participant F is marginally higher, while those for other participants are significantly higher. For all participants, sEMG amplitudes in AST1.5y conditions are all significantly higher than those in AST0 conditions. This suggests that voluntary ankle dorsiflexion movement was induced with dorsiflexion assistance delay time equal to or larger than y ms, which successfully identifies an optimal assistance timing. Meanwhile, sEMG amplitudes in AST0.5y conditions for Participants B and E are also significantly larger than those in AST0, while the other participants' results show no significant differences. 

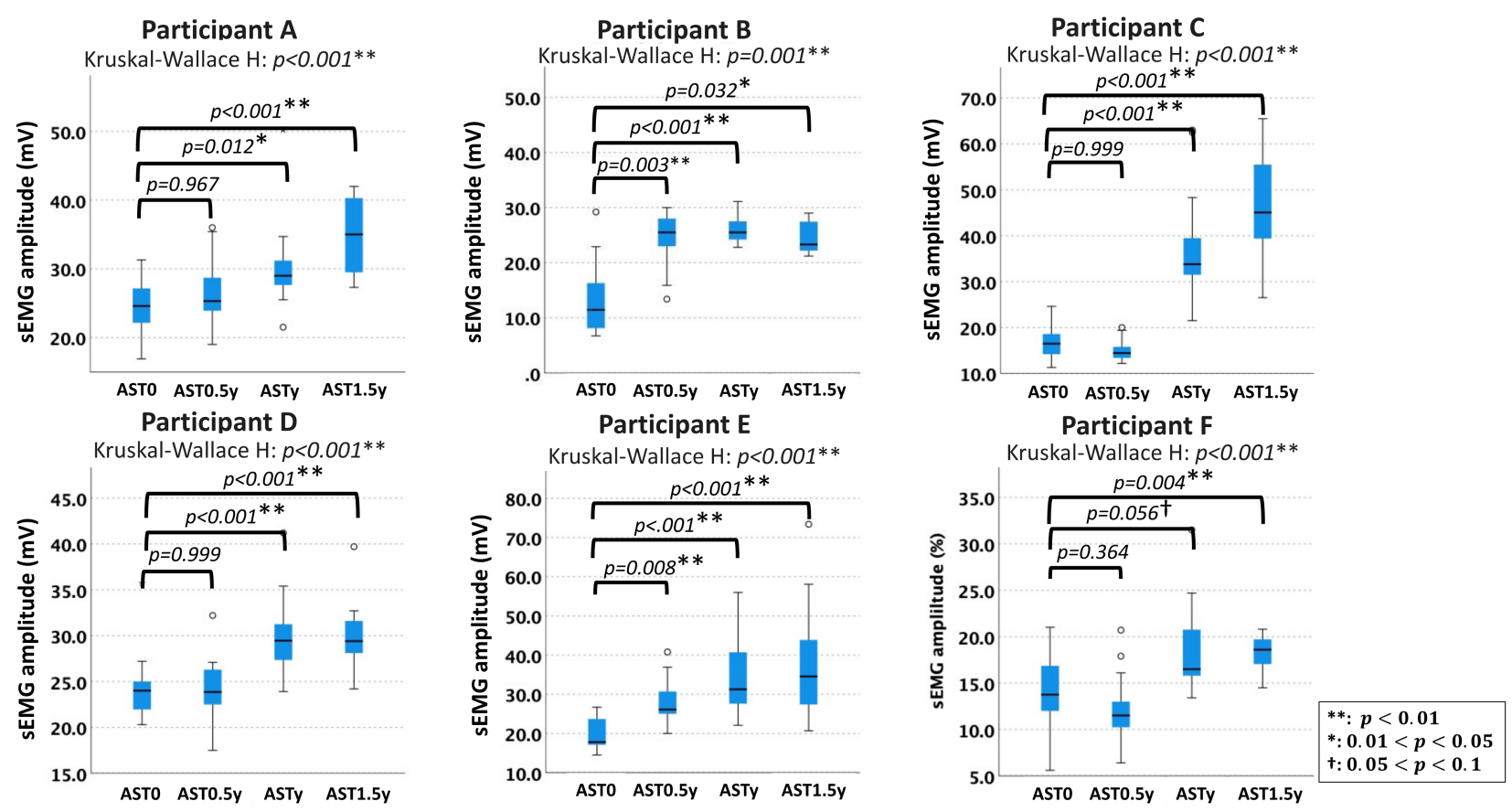

Figure 8. sEMG amplitude results.

\subsection{The Kinematic Results}

Figures 9 and 10 show the extent of observed compensatory movements, i.e., swing width and hip hike. Results of the Kruskal-Wallis H tests show overall significant differences between all groups. Regarding pair-wise results, only Participant A's hip hike in AST0 was marginally smaller than that in the SCG condition, while the other participants' swing width and hip hike results in AST0, AST0.5y, and ASTy are all significantly lower than those in the SCG condition. This indicates successful suppression of compensatory movements with a dorsiflexion assistance delay shorter than the identified y. On the other hand, some participants' swing widths or hip hikes in AST1.5y show no significant difference when compared to those in the SCG condition.

Participant A

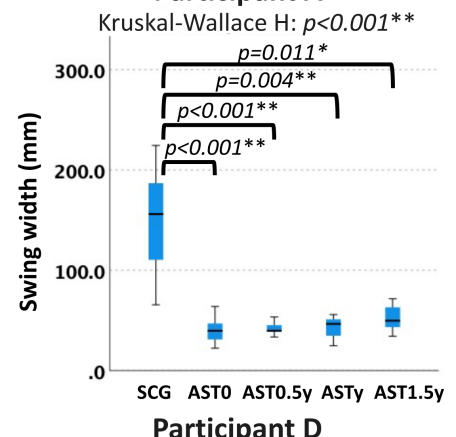

Participant D

Kruskal-Wallace H: $p<0.001^{* *}$

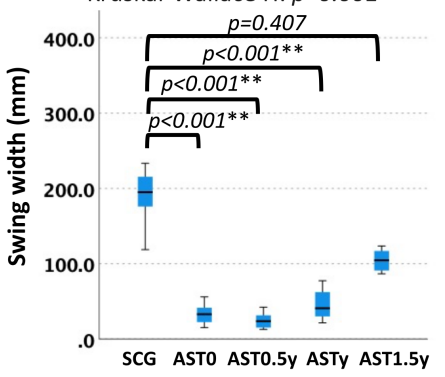

Participant B

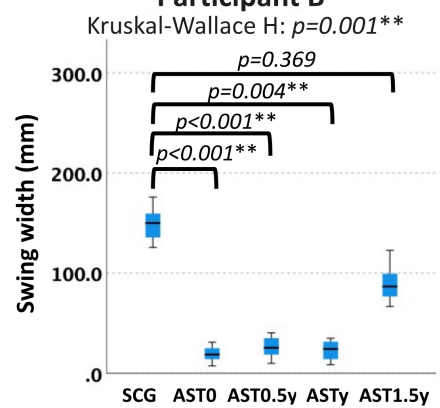

Participant E

Kruskal-Wallace $\mathrm{H}: p<0.001^{* *}$

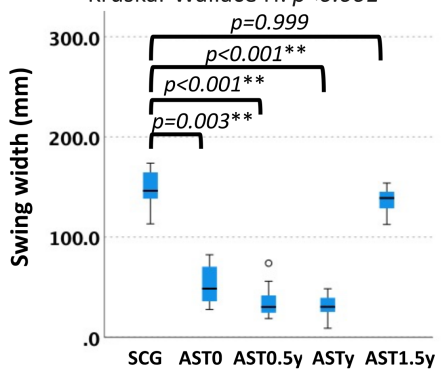

Participant C

Kruskal-Wallace $\mathrm{H}: p<0.001^{* *}$

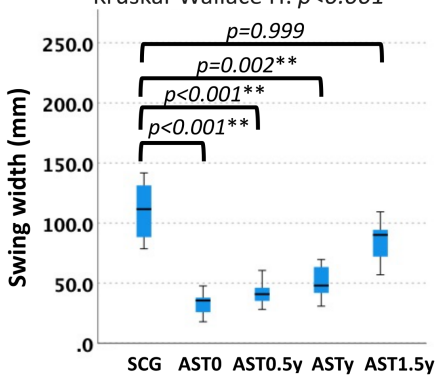

Participant $\mathbf{F}$

Kruskal-Wallace $\mathrm{H}: p<0.001 * *$

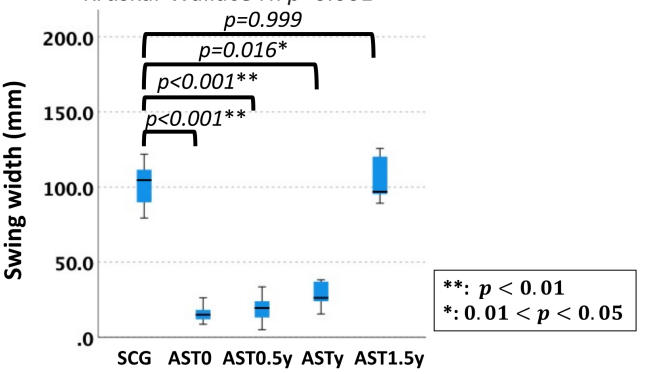

Figure 9. Swing width results. 

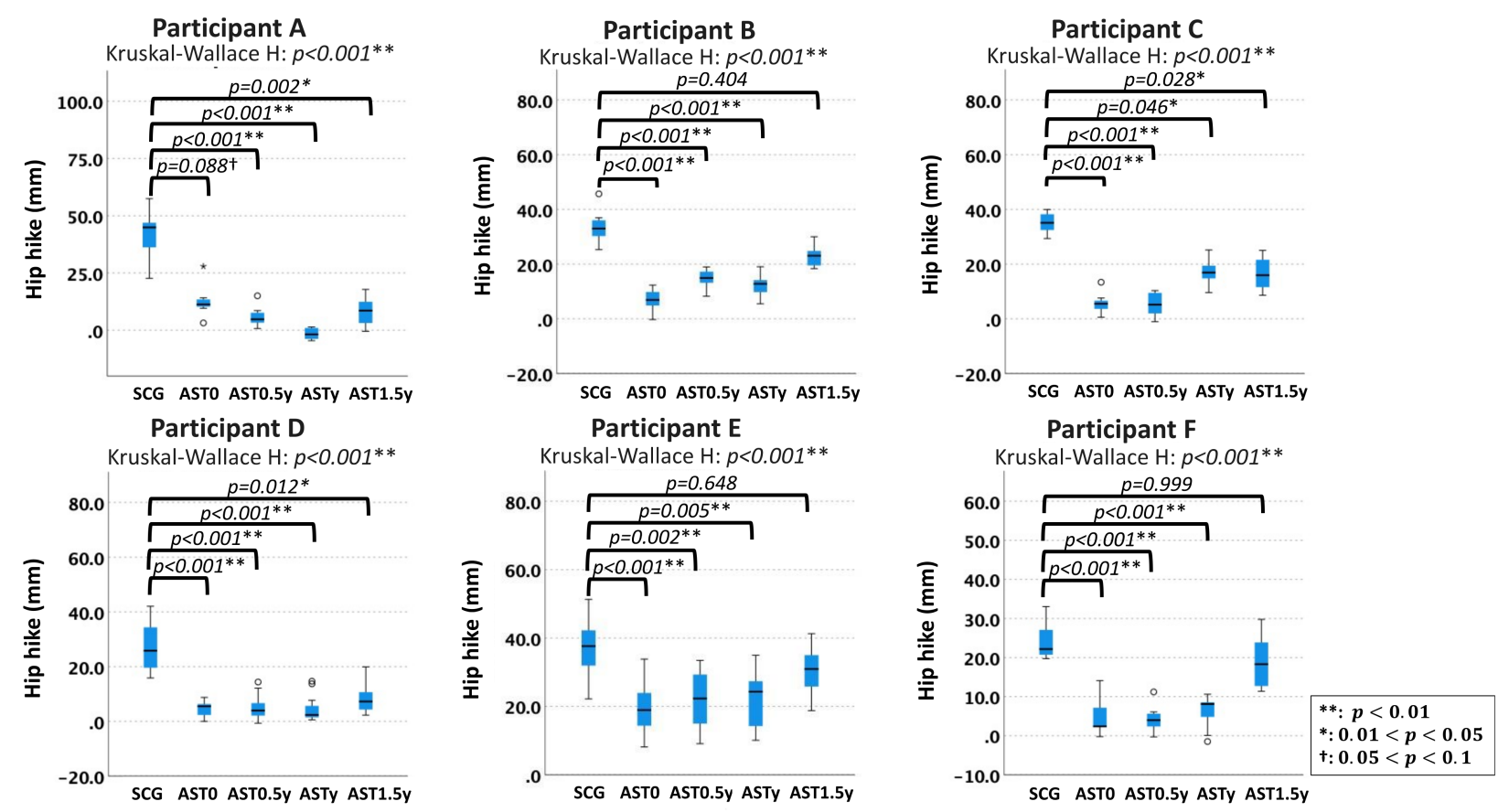

Figure 10. Hip hike results.

Results of limb shortening and ankle dorsiflexion angle are shown in Figures 11 and 12. All results of the Kruskal-Wallis $\mathrm{H}$ tests show highly significant differences between all groups. Limb shortening in AST0, AST0.5y, and ASTy are all significantly larger than those in the SCG condition, which indicates improvement in limb shortening with a dorsiflexion assistance timing equal or shorter than the identified delay time. On the other hand, only Participant B's limb shortening in AST1.5y is significantly higher than that in SCG, while the other participants' results did not show significant differences. For ankle dorsiflexion angle, result patterns for all participants vary. Participants B and C's ankle dorsiflexion angles in ASTy have no significant difference compared with those in SCG, which is not as expected.
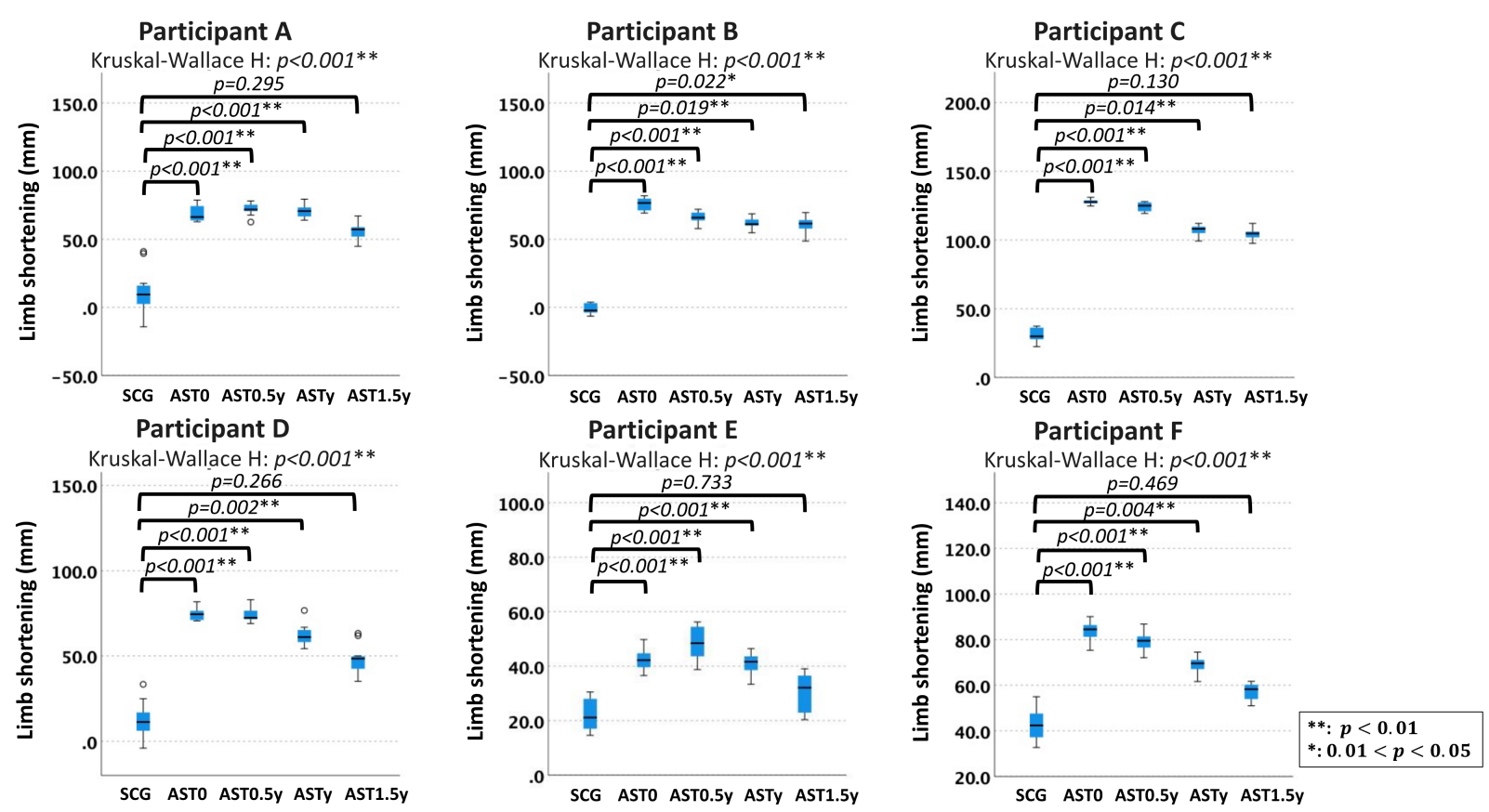

Figure 11. Limb shortening results. 

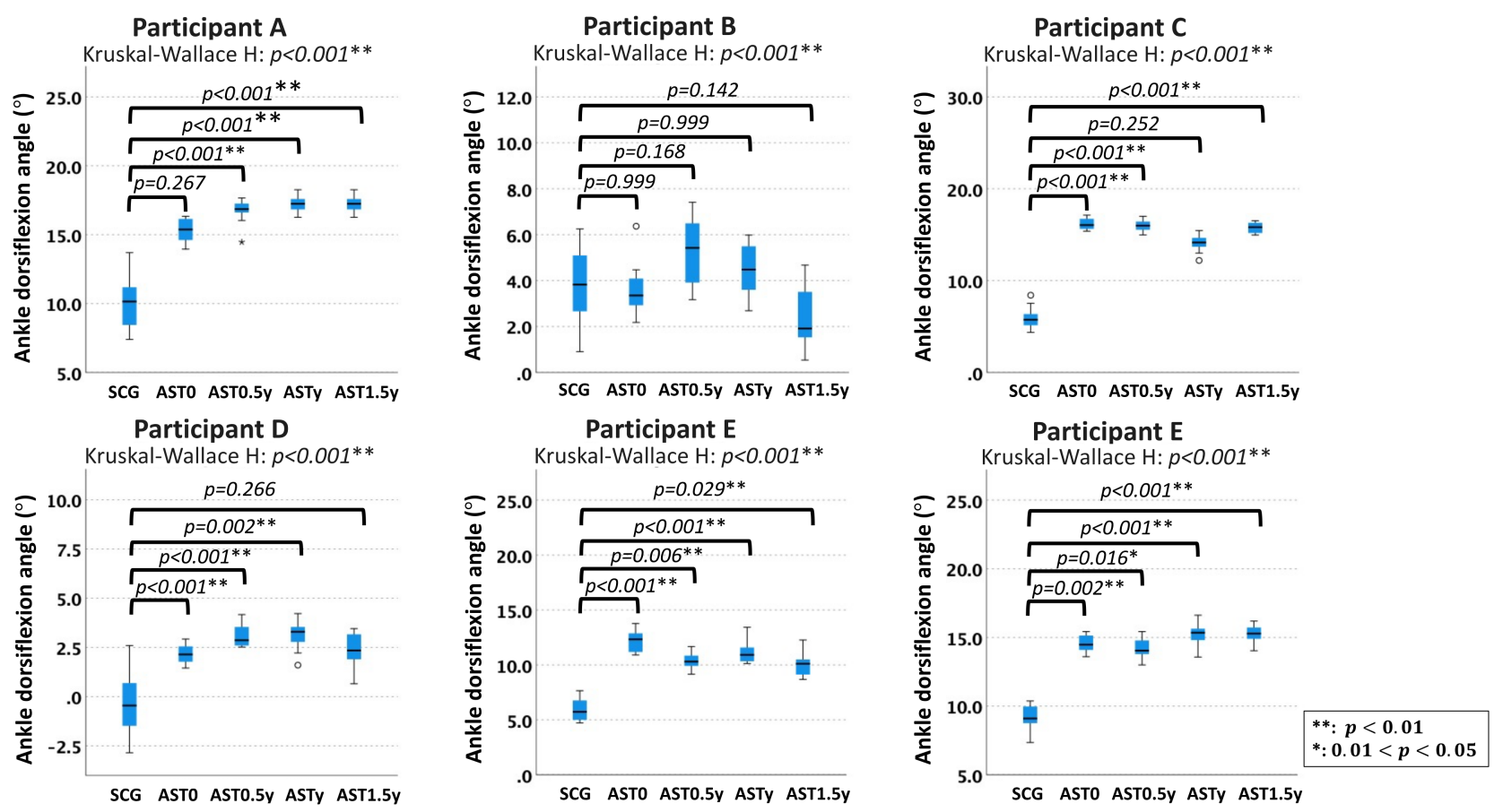

Figure 12. Ankle dorsiflexion angle in the swing phase.

\section{Discussion}

In our research, we have identified optimal delayed dorsiflexion assistance timing after toe-off and conducted an experiment to verify the assistance effects on healthy participants with restricted swing-phase dorsiflexion. Our previous study indicated a trade-off relation between ensuring voluntary efforts and preventing compensatory movements [26]. Later swing-phase dorsiflexion assistance timing could induce voluntary effort but could also exacerbate compensatory movements. On the other hand, earlier assistance could prevent compensatory movements but could hinder voluntary efforts. Thus, we assumed that there is an assistance timing that could induce voluntary efforts while suppressing compensatory movements, which we sought to identify.

With the identified assistance delay (ASTy), the sEMG amplitude results showed an improvement in voluntary effort during swing-phase dorsiflexion, and the swing width and hip hike results show suppression of compensatory movements. Some differences were observed with shorter (AST0.5y) and longer assistance delays (AST1.5y). sEMG amplitude results with AST0.5y for Participants C and D also significantly increased compared with those with AST0. Meanwhile, the extent of compensatory movements with AST1.5y was also significantly suppressed when compared with those in SCG for some participants, especially Participant A. Figure 13 explains the assumed reason for these results with shorter and longer assistance delays. Instead of a specific suitable assistance timing, there is a range of ideal delay times for dorsiflexion assistance. For Participants C and D, their sEGM amplitudes increased with not only ASTy but also AST0.5y, which indicates that assistance delay times $0.5 \mathrm{y}$ and $\mathrm{y}$ are both in the range of suitable dorsiflexion delay. On the other hand, Participant A's swing-phase and hip hike were suppressed with both ASTy and AST1.5y, which indicates that $\mathrm{y}$ and 1.5y assistance delay times were both suitable for Participant A. It should also be noted that Participant F's sEMG result with ASTy was marginally increased compared to AST0. This suggests that the identified timing for Participant $\mathrm{F}$ is at the lower bound of the suitable timing zone, also shown in Figure 13. Despite such differences, our goal of inducing voluntary dorsiflexion and suppressing compensatory movement with identified assistance delay was met. 


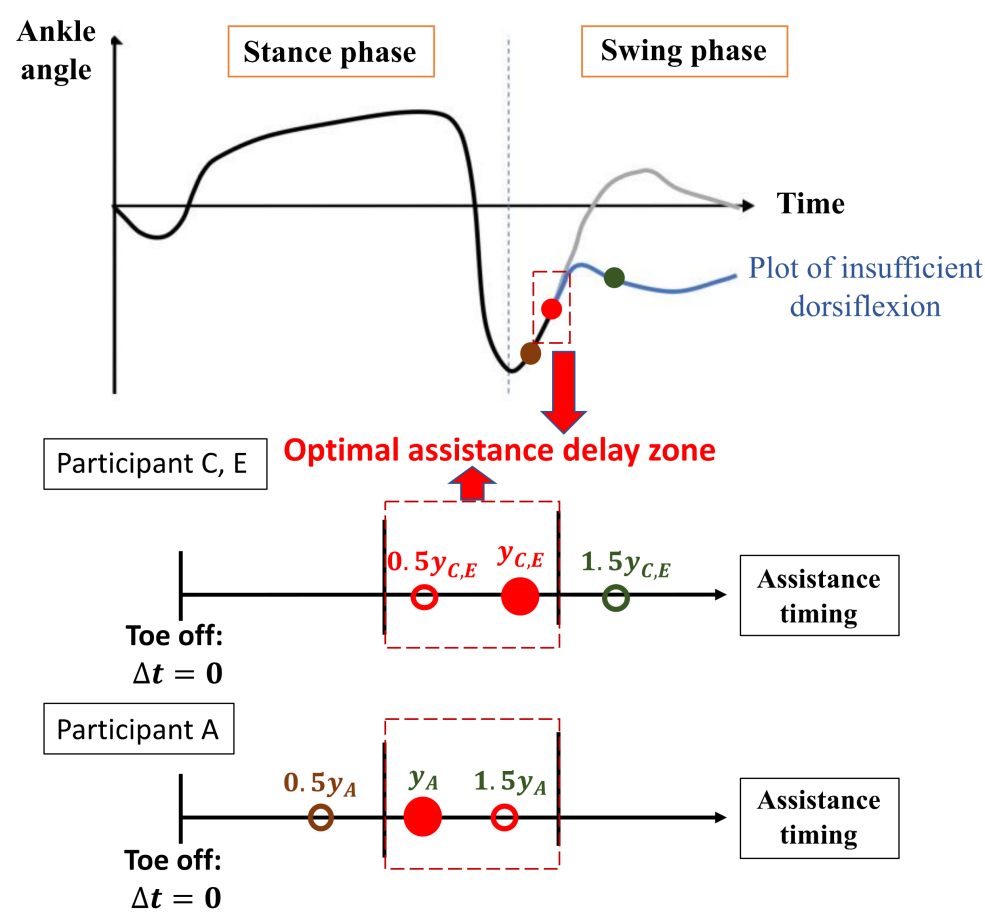

Figure 13. Indication of identified assistance timing of the participants.

Limb shortening results are also consistent among participants, and those with the identified assistance delay time (ASTy) were significantly larger when compared to the restricted dorsiflexion walking condition (SCG). This is consistent with an observed tradeoff between limb shortening and compensatory movements from a related study [6]. The study also notes that ankle angle is one of the factors that affects limb shortening. However, results of our experiment are not consistent with that theory. For Participants B and C, their swing-phase ankle dorsiflexion angles in ASTy had no significant difference compared to those in SCG. Since limb shortening could also be affected by knee movement, we reanalyzed participants' knee flexion angles in the swing phase. Two examples of knee flexion angle from Participants B and E are shown in Figure 14. Both data sets show knee flexion differences between conditions, which is expected. Past studies have also pointed out that resistance or assistance in ankle movements also affects knee and hip kinematics [37,38]. However, Participant B's knee flexion angle in SCG is $18.95^{\circ} \pm 2.32^{\circ}$, which is abnormally small when compared with the average angle of a healthy person, between $45^{\circ}$ and $55^{\circ}$ [39]. Therefore, it is clear that Participant B did not only exhibit circumduction and hip hiking but also decreased knee flexion during the swing phase. Therefore, Participant B's limb shortening might be dominated by knee flexion instead of ankle dorsiflexion during the swing phase.
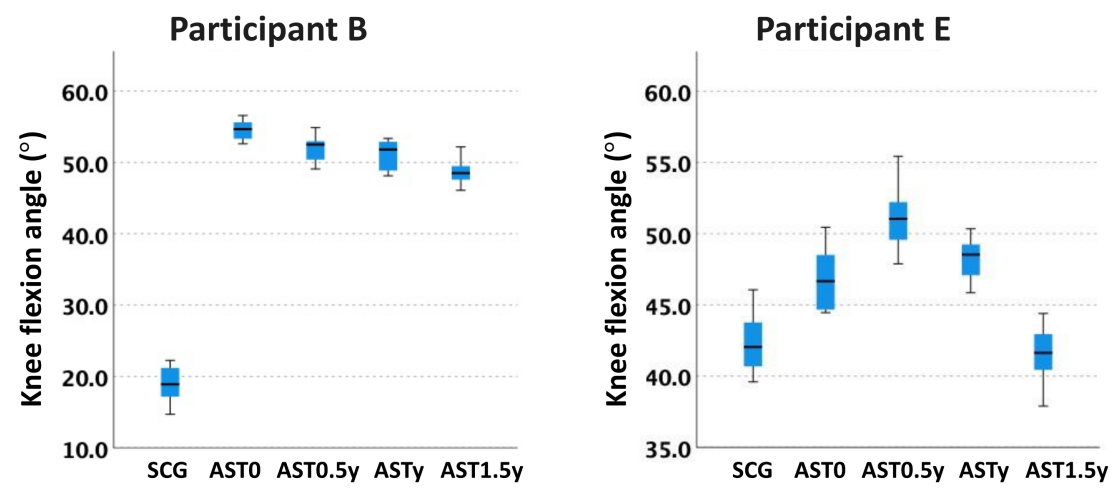

Figure 14. Results of knee flexion angle in swing phase for Participants B and E. 
Limitations of this research include using healthy participants with dorsiflexionrestricted gaits. No other restrictions were applied on the participants. This means the observed gait patterns were only due to restriction and assistance on swing-phase dorsiflexion movement. On the other hand, in addition to insufficient ankle dorsiflexion, stroke survivors could also suffer from weak knees and hips, sensory paralysis, muscle spasticity, and other injuries [40-42]. These could further affect diversity of their pathological gait patterns. One such example is insufficient knee flexion in the swing phase which could especially affect the results of compensatory movements due to its larger contribution to limb shortening compared with swing-phase dorsiflexion [6]. Thus, knee movement assistance might also be required with our time delay dorsiflexion assistance. Another example is sole sensory paralysis [43]. The method in this study assumed that a stroke survivor could dorsiflex upon toe-off to a certain extent. Due to sensory paralysis at the affected side, a stroke patient could not sense toe-off and thus could not dorsiflex. In this case, another assistance device such us our perception-empathy biofeedback system that provides biofeedback of foot pressure should be used $[30,31]$. The other limitation is identifying assistance delay time in milliseconds. Participants of the present research are healthy, so their gait periods are consistent. The ultimate subject of our identification method is stroke survivors with pathological gaits. Although our targets are stroke survivors who can already walk independently, an unstable gait period could still be expected. Moreover, their gaits may also change when under our system's intervention and under different assistance timings.

Based on the stated limitations, future work includes a practical study of stroke survivors and development of an adaptive control method that could modify assistance timing based on patients' gait conditions in real time. A new proposal of a gait assistance method might also be required due to their disabilities in addition to ankle weakness. Although there are still required improvements, we believe that the present research provides a method to induce swing-phase dorsiflexion movement and suppress compensatory movements at the same time.

\section{Conclusions}

In this research, we derived a method to identify suitable swing-phase dorsiflexion assistance timing that simultaneously improves voluntary efforts and suppresses compensatory movements. Via a multiple linear regression analysis on ankle data of stroke patients whose paralyzed sides suffer insufficient dorsiflexion in the swing phase, the identification equation was derived. With this method, an experiment was conducted on healthy participants with dorsiflexion-restricted gaits. With the identified assistance timing, a significant improvement in voluntary effort due to an increase in limb shortening was observed. Meanwhile, improvements in limb shortening and suppression of compensatory movements such as circumduction and hip hiking were also observed. Limitations of this study include using healthy people as participants. For stroke patients, results might differ due to other problems such as sensory paralysis and varied gait cycle in every step. Applying other assistance strategies along with the proposed method might be required in practical cases. However, this research provides a potential method for accelerated gait rehabilitation assistance. Impedance control is mostly applied by motor-driven systems to enhance voluntary effort, while the method we proposed could also be potential used for pneumatically driven systems. Future work includes improvement of the assistance algorithm and a practical study on stroke survivors.

Author Contributions: Conceptualization, methodology, formal analysis, visualization, and writing—original draft preparation, J.-C.H.; investigation and writing—review and editing, K.Y.; resources and supervision, H.O.; supervision, H.I. All authors have read and agreed to the published version of the manuscript.

Funding: This research was funded by JSPS KAKENHI Grant, Grant Number JP26289068, MIKIYA Science and Technology Foundation, and Waseda University. 
Institutional Review Board Statement: The study was conducted according to the guidelines of the Declaration of Helsinki and approved by the Ethics Committee of Waseda University (No. 2017-044).

Informed Consent Statement: Informed consent was obtained from all subjects involved in the study.

Data Availability Statement: All data are presented in the manuscript.

Acknowledgments: The authors would like to thank the participants for their help in the experiment.

Conflicts of Interest: The authors declare no conflict of interest.

\section{References}

1. Stroke, Cerebrovascular Accident. Available online: http://www.emro.who.int/health-topics/stroke-cerebrovascular-accident/ index.html (accessed on 11 January 2022).

2. Krishnamurthi, R.V.; Ikeda, T.; Feigin, V.L. Global, regional and country-specific burden of ischaemic stroke, intracerebral haemorrhage and subarachnoid haemorrhage: A systematic analysis of the global burden of disease study 2017. Neuroepidemiology 2020, 54, 171-179. [CrossRef] [PubMed]

3. Bruni, M.F.; Melegari, C.; De Cola, M.C.; Bramanti, A.; Bramanti, P.; Calabrò, R.S. What does best evidence tell us about robotic gait rehabilitation in stroke patients: A systematic review and meta-analysis. J. Clin. Neurosci. 2018, 48, 11-17. [CrossRef] [PubMed]

4. Otter, A.; Geuruts, A.; Mulder, T.; Duysens, J. Abnormalities in the temporal patterin of lower extremity muscle activity in hemiparetic gait. Gait Posture 2007, 25, 342-352. [CrossRef] [PubMed]

5. Perera, C.K.; Gopalai, A.A.; Ahmad, S.A.; Gouwanda, D. Muscles affecting minimum toe clearance. Front. Public Health 2021, 9, 612064. [CrossRef] [PubMed]

6. Matsuda, F.; Mukaino, M.; Ohtsuka, K.; Tanikawa, H.; Tsuchiyama, K.; Teranishi, T.; Kanada, Y.; Kagaya, H.; Saitoh, E. Biomechanical factors behind toe clearance during the swing phase in hemiparetic patients. Top. Stroke Rehabil. 2017, 24, 177-182. [CrossRef]

7. Gil-Castillo, J.; Alnajjar, F.; Koutsou, A.; Torricelli, D.; Moreno, J.C. Advances in neuroprosthetic management of foot drop: A review. J. Neuroeng. Rehabil. 2020, 17, 46. [CrossRef]

8. Best, R.; Begg, R. A method for calculating the probability of tripping while walking. J. Biomech. 2008, 41, 1147-1151. [CrossRef]

9. Balaban, B.; Tok, F. Gait disturbances in patients with stroke. PMER 2014, 6, 635-642.

10. Matsuda, F.; Mukaino, M.; Ohtsuka, K.; Tanikawa, H.; Tsuchiyama, K.; Teranishi, T.; Kanada, Y.; Kagaya, H.; Saitoh, E. Analysis of strategies used by hemiplegic stroke patients to achieve toe clearance. Jpn. J. Compr. Rehabil. Sci. 2016, 7, 111-118.

11. Hussain, S.; Jamwal, P.K.; Vliet, P.V.; Brown, N.A.T. Robot assisted ankle neuro-rehabilitation: State of the art and future challenges. Expert Rev. Neurother. 2021, 21, 111-121. [CrossRef]

12. Alnajjar, F.; Zaier, R.; Khalid, S.; Gochoo, M. Trends and technologies in rehabilitation of foot drop: A systematic review. Expert Rev. Med. Devices 2021, 18, 31-46. [CrossRef] [PubMed]

13. Oba, T.; Kadone, H.; Hassan, M.; Suzuki, K. Robotic ankle-foot orthosis with a variable viscosity link using MR fluid. IEEE ASME Trans. Mechatron. 2019, 24, 495-504. [CrossRef]

14. Nakagawa, K.; Tomoi, M.; Higashi, K.; Utsumi, S.; Kawano, R.; Tanaka, E.; Kurisu, K.; Yuge, L. Short-term effect of a close-fitting type of walking assistive device on spinal cord reciprocal inhibition. J. Clin. Neurosci. 2020, 77, 142-147. [CrossRef] [PubMed]

15. Jamwal, P.K.; Hussain, S.; Ghayesh, M.H. Robotic orthoses for gait rehabilitation: An overview of mechanical design and control strategies. Proc. Inst. Mech. Eng. H 2020, 234, 444-457. [CrossRef] [PubMed]

16. Hidayah, R.; Bishop, L.; Jin, X.; Chamarthy, S.; Stein, J.; Agrawal, S.K. Gait adaptation using a cable-driven active leg exoskeleton (C-ALEX) with post-stroke participants. IEEE Trans. Neural Syst. Rehabil. Eng. 2020, 28, 1984-1993. [CrossRef]

17. Van Kammen, K.; Boonstra, A.M.; van der Woude, L.H.V.; Visscher, C.; Reinders-Messelink, H.A.; den Otter, R. Lokomat guided gait in hemiparetic stroke patients: The effects of training parameters on muscle activity and temporal symmetry. Disabil. Rehabil. 2020, 42, 2977-2985. [CrossRef]

18. Lotze, M.; Braun, C.; Birbaumer, N.; Anders, S.; Cohen, L.G. Motor learning elicited by voluntary drive. Brain 2003, 126, 866-872. [CrossRef]

19. Adiputra, D.; Nazmi, N.; Bahiuddin, I.; Ubaidillah, U.; Imaduddin, F.; Abdul Rahman, M.A.; Mazlan, S.A.; Zamzuri, H. A review on the control of the mechanical properties of ankle foot orthosis for gait assistance. Actuators 2019, 8, 10. [CrossRef]

20. Forrester, L.W.; Roy, A.; Krebs, H.I.; Macko, R.F. Ankle training with a robotic device improves hemiparetic gait after a stroke. Neurorehabil. Neural Repair 2011, 25, 369-377. [CrossRef]

21. Chaparro-Rico, B.D.M.; Cafolla, D. Test-retest, inter-rater and intra-rater reliability for spatiotemporal gait parameters using SANE (an eaSy gAit aNalysis systEm) as measuring instrument. Appl. Sci. 2020, 10, 5781. [CrossRef]

22. Chaparro-Rico, B.D.M.; Cafolla, D.; Tortola, P.; Galardi, G. Assessing stiffness, joint torque and ROM for paretic and non-paretic lower limbs during the subacute phase of stroke using lokomat tools. Appl. Sci. 2020, 10, 6168. [CrossRef]

23. Roy, A.; Krebs, H.; Williams, D.; Bever, C.; Forrester, L.; Macko, R.; Hogan, N. Robot-aided nerorehabilitation: A novel robot for ankle rehabilitation. IEEE Trans. Robot. 2009, 25, 569-582. [CrossRef] 
24. Cruz, T.H.; Dhaher, Y.Y. Impact of ankle-foot orthosis on frontal plane behaviors post-stroke. Gait Posture 2009, 30, 312-316. [CrossRef] [PubMed]

25. Hong, J.C.; Cheng, H.; Yasuda, K.; Ohashi, H.; Iwata, H. Effects of assisted dorsiflexion timing on voluntary efforts and compensatory movements: A feasibility study in healthy participants. IEEE Trans. Neural Syst. Rehabil. Eng. 2021, 29, $2222-2231$. [CrossRef]

26. Hong, J.-C.; Suzuki, S.; Fukushima, Y.; Yasuda, K.; Ohashi, H.; Iwata, H. Development of high-dorsiflexion assistive robotic technology for gait rehabilitation. In Proceedings of the 2018 IEEE International Conference on Systems, Man, and Cybernetics (SMC), Miyazaki, Japan, 7-10 October 2018; pp. 3801-3806.

27. Hong, J.-C.; Cheng, H.; Hayashi, Y.; Yasuda, K.; Ohashi, H.; Iwata, H. Evaluation of the effect of high-dorsiflexion assistive robotic technology on voluntary ankle movement. In Proceedings of the 2020 8th IEEE RAS/EMBS International Conference for Biomedical Robotics and Biomechatronics (BioRob), New York, NY, USA, 29 November-1 December 2020; pp. 25-29.

28. Tanaka, E.; Muramatsu, K.; Watanuki, K.; Saegusa, S.; Yuge, L. Walking assistance apparatus enabled for neuro-rehabilitation of patients and its effectiveness. Mech. Eng. Lett. 2015, 1, 00530. [CrossRef]

29. Hong, J.-C.; Tanaka, G.; Yasuda, K.; Ohashi, H.; Iwata, H. Identifying spring coefficient for assisting hemiplegic Patient's heel rocker function: A feasibility study. In Proceedings of the 2020 IEEE International Conference on Systems, Man, and Cybernetics (SMC), Toronto, ON, Canada, 11-14 October 2020; pp. 738-743.

30. Hayashi, Y.; Yasuda, K.; Kitaji, K.; Harashima, H.; Iwata, H. A haptic-based perception-empathy biofeedback device that supplements foot pressure pattern during gait in stroke patients. In Proceedings of the 2019 IEEE/SICE International Symposium on System Integration (SII), Paris, France, 14-16 January 2019; pp. 124-128.

31. Yasuda, K.; Saichi, K.; Kaibuki, N.; Harashima, H.; Iwata, H. Haptic-based perception-empathy biofeedback system for balance rehabilitation in patients with chronic stroke: Concepts and initial feasibility study. Gait Posture 2018, 62, 484-489. [CrossRef] [PubMed]

32. Yasuda, K.; Hayashi, Y.; Tawara, A.; Iwata, H. Development of a vibratory cueing system using an implicit method to increase walking speed in patients with stroke: A proof-of-concept study. ROBOMECH J. 2020, 7, 1. [CrossRef]

33. Ling, J.; Yasuda, K.; Hayashi, Y.; Imamura, S.; Iwata, H. Development of a vibrotactile cueing device that implicitly increases walking speed during gait training in stroke patients: An observational case series study. J. Med. Eng. Technol. 2021, 46, 1-7. [CrossRef]

34. Donath, L.; Faude, O.; Lichtenstein, E.; Nüesch, C.; Mündermann, A. Validity and reliability of a portable gait analysis system for measuring spatiotemporal gait characteristics: Comparison to an instrumented treadmill. J. Neuroeng. Rehabil. 2016, 13, 6. [CrossRef]

35. Moreira, L.; Figueiredo, J.; Fonseca, P.; Vilas-Boas, J.P.; Santos, C.P. Lower limb kinematic, kinetic, and EMG data from young healthy humans during walking at controlled speeds. Sci. Data 2021, 8, 103. [CrossRef]

36. Farfán, F.D.; Politti, J.D.; Felice, C.J. Evaluation of EMG processing techniques using information theory. Biomed. Eng. Online 2010, 9, 1. [CrossRef] [PubMed]

37. Sekiguchi, Y.; Owaki, D.; Honda, K.; Fukushi, K.; Hiroi, N.; Nozaki, T.; Izumi, S.I. Ankle-foot orthosis with dorsiflexion resistance using spring-cam mechanism increases knee flexion in the swing phase during walking in stroke patients with hemiplegia. Gait Posture 2020, 81, 27-32. [CrossRef] [PubMed]

38. Totah, D.; Menon, M.; Jones-Hershinow, C.; Barton, K.; Gates, D.H. The impact of ankle-foot orthosis stiffness on gait: A systematic literature review. Gait Posture 2019, 69, 101-111. [CrossRef] [PubMed]

39. Perry, J.; Burnfield, J. Gait Analysis: Normal and Pathological Function, 2nd ed.; SLACK Incorporated: West Deptford, NJ, USA, 2010.

40. Chae, C.S.; Jun, J.H.; Im, S.; Jang, Y.Y.; Park, G.Y. Effectiveness of hydrotherapy on balance and paretic knee strength in patients with stroke. Am. J. Phys. Med. Rehabil. 2020, 99, 409-419. [CrossRef] [PubMed]

41. Dubey, L.; Karthikbabu, S.; Mohan, D. Effects of pelvic stability training on movement control, hip muscles strength, walking speed and daily activities after stroke: A randomized controlled trial. Ann. Neurosci. 2018, 25, 80-89. [CrossRef]

42. Gittins, M.; Lugo-Palacios, D.; Vail, A.; Bowen, A.; Paley, L.; Bray, B.; Tyson, S. Stroke impairment categories: A new way to classify the effects of stroke based on stroke-related impairments. Clin. Rehabil. 2021, 35, 446-458. [CrossRef]

43. Pumpa, L.U.; Cahill, L.S.; Carey, L.M. Somatosensory assessment and treatment after stroke: An evidence-practice gap. Aust. Occup. Ther. J. 2015, 62, 93-104. [CrossRef] 